\title{
The Green's function of a class of two-term fractional differential equation boundary value problem and its applications
}

\section{Yongqing Wang ${ }^{1 *}$}

\section{"Correspondence:}

wyqing9801@163.com

'School of Mathematical Sciences, Qufu Normal University, Qufu, P.R. China

\section{Springer}

\begin{abstract}
In this paper, we consider a Riemann-Liouville type two-term fractional differential equation boundary value problem. Some positive properties of the Green's function are deduced by using techniques of analysis. As application, we obtain the existence and multiplicity of positive solutions for a fractional boundary value problem under conditions that the nonlinearity $f(t, x)$ may change sign and may be singular at $t=0,1$ and $x=0$, and we also obtain the uniqueness results of positive solution for a singular problem by means of the monotone iterative technique.
\end{abstract}

Keywords: Two-term fractional differential equation; Boundary value problems; Green's function; Positive solution; Singularity

\section{Introduction}

In this paper, we study properties of the Green's function of the following two-term fractional differential equation boundary value problem (FBVP):

$$
\left\{\begin{array}{l}
-D_{0+}^{\alpha} u(t)+a u(t)=y(t), \quad 0<t<1, \\
u(0)=u^{\prime}(0)=0, \quad u(1)=0,
\end{array}\right.
$$

where $2<\alpha<3, a>0, D_{0+}^{\alpha}$ is the standard Riemann-Liouville derivative.

During the past decades, much attention has been paid to the study of fractional differential equations (FDEs) due to the more accurate effect in describing important phenomena in biology, engineering, and so on. It has been proved that a multi-term FDE can be used to describe various types of visco-elastic damping $[1,2]$. Most of the model equations proposed can be expressed by the linear form

$$
\left[D^{\alpha_{N}}+a_{N-1} D^{\alpha_{N-1}}+\cdots+a_{1} D^{\alpha_{1}}+a_{0} D^{0}\right] x(t)=f(t),
$$

where $a_{i} \in \mathbf{R}, i=0,1, \ldots, N-1$, equipped with initial conditions (see [3-7] and the references therein). For example, Elshehawey et al. [5] considered the endolymph equation

$$
D^{2} x(t)+a_{1} D x(t)+a_{2} D^{\frac{1}{2}} x(t)+a_{3} x(t)=-f(t)
$$

(c) The Author(s) 2020. This article is licensed under a Creative Commons Attribution 4.0 International License, which permits use, sharing, adaptation, distribution and reproduction in any medium or format, as long as you give appropriate credit to the original author(s) and the source, provide a link to the Creative Commons licence, and indicate if changes were made. The images or other third party material in this article are included in the article's Creative Commons licence, unless indicated otherwise in a credit line to the material. If material is not included in the article's Creative Commons licence and your intended use is not permitted by statutory regulation or exceeds the permitted use, you will need to obtain permission directly from the copyright holder. To view a copy of this licence, visit http://creativecommons.org/licenses/by/4.0/. 
which can be used to describe the response of the semicircular canals to the angular acceleration.

Recently, many authors have focused on the existence of solutions to nonlinear FBVPs by using the techniques of nonlinear analysis such as fixed point theorems, Leray-Schauder theory, etc. (see [8-32]). Since only positive solutions are meaningful in most practical problems, the existence of positive solutions for FBVPs has particularly attracted a great deal of attention, e.g., the nonlocal FBVPs [10, 22, 25], singular FBVPs [16, 21, 28], semipositone FBVPs $[15,18,27]$.

It is known that the cone which usually depends on the positive properties of the Green's function plays a very important role in discussing positive solutions. When $1<\alpha<2$, Jiang and Yuan [14] obtained some properties of the Green's function for the FDE:

$$
D_{0+}^{\alpha} u(t)+f(t, u(t))=0, \quad 0<t<1,
$$

with Dirichlet type boundary value condition. Xu and Fei [30] investigated (2) with threepoint boundary value condition. In [19], we established some new positive properties of the corresponding Green's function for (2) with multi-point boundary value condition. When $\alpha>2$, Zhang et al. [27, 28] obtained triple positive solutions for (2) with conjugate type integral conditions by employing height functions on special bounded sets which were derived from properties of the Green's function.

While there are a lot of works dealing with multi-term FDEs with initial conditions, the results dealing with boundary value problems of multi-term FDEs are relatively scarce. For some recent literature on Caputo type multi-term FBVPs, we mention the papers [8,9] and the references therein. In [20], we established some new positive properties of the Green's function for the Riemann-Liouville type FBVP, in which the linear operator contains two terms:

$$
\left\{\begin{array}{l}
-D_{0+}^{\alpha} u(t)+b u(t)=f(t, u(t)), \quad 0<t<1, \\
u(0)=0, \quad u(1)=0,
\end{array}\right.
$$

where $1<\alpha<2, b>0$. As application, the existence and uniqueness of positive solution are obtained under singular conditions.

Inspired by the above work, in this paper, we aim to deduce some positive properties of the Green's function for FBVP (1). As application, we investigate the existence and multiplicity of positive solutions for a singular FBVP with changing sign nonlinearity, and we also consider the uniqueness results of positive solution for a singular FBVP. Compared with the existing works, this paper has the following features. Firstly, the fractional derivative discussed in this paper is the standard Riemann-Liouville derivative, which is different from $[8,9]$, and the linear operator of the FBVP we are considered with contains two terms, which is different from $[14,19,27,28,30]$; in other words, we discuss different problem which has been seldom studied before. Secondly, some meaningful properties of the Green's function for the case that $2<\alpha<3$ are established; this is different from [20] since Ref. [20] considered the case that $1<\alpha<2$. Thirdly, we consider a multiplicity of positive solutions under conditions that the nonlinearity $f(t, x)$ may change sign and possess singularity at $x=0$; this is different from $[15,18]$. It should be noted that there are relatively few results on multiple solutions for FBVPs under this circumstance, not to 
mention two-term FBVPs. Finally, we obtain the uniqueness results of positive solution for a singular two-term FBVP by means of the monotone iterative technique, and the rate of convergence for the iterative sequence is considered.

\section{Basic definitions and preliminaries}

Definition 2.1 ([33]) The fractional integral of a function $u:(0,+\infty) \rightarrow R$ is given by

$$
I_{0+}^{\alpha} u(t)=\frac{1}{\Gamma(\alpha)} \int_{0}^{t}(t-s)^{\alpha-1} u(s) d s
$$

provided that the right-hand side is point-wise defined on $(0,+\infty)$.

Definition 2.2 ([33]) The Riemann-Liouville fractional derivative of a function $u$ : $(0,+\infty) \rightarrow R$ is given by

$$
D_{0+}^{\alpha} u(t)=\frac{1}{\Gamma(n-\alpha)}\left(\frac{d}{d t}\right)^{n} \int_{0}^{t}(t-s)^{n-\alpha-1} u(s) d s,
$$

where $n=[\alpha]+1,[\alpha]$ denotes the integer part of number $\alpha$, provided that the right-hand side is point-wise defined on $(0,+\infty)$.

For convenience, we introduce the following notations:

$$
\begin{aligned}
& h(x)=\sum_{k=0}^{+\infty} \frac{(k \alpha+\alpha-2)(k \alpha+\alpha-3) x^{k}}{\Gamma(k \alpha+\alpha)}, \\
& k(s)=(1-s)^{\alpha-2}-s, \\
& g(t)=t^{\alpha-1} E_{\alpha, \alpha}\left(a t^{\alpha}\right), \\
& g_{1}(t)=t^{\alpha-2} E_{\alpha, \alpha}\left(a t^{\alpha}\right),
\end{aligned}
$$

where

$$
E_{\alpha, \alpha}(x)=\sum_{k=0}^{+\infty} \frac{x^{k}}{\Gamma((k+1) \alpha)}
$$

is the Mittag-Leffler function.

It is clear that $h(x)$ is strictly increasing on $[0,+\infty), h(0)<0$, and

$$
\lim _{x \rightarrow+\infty} h(x)=+\infty
$$

Therefore, $h(x)$ has a unique positive root $a^{*}$, that is, $h\left(a^{*}\right)=0$.

Throughout this paper, we always assume that the following assumption holds:

$\left(H_{1}\right) a \in\left(0, a^{*}\right]$ is a constant.

Lemma 2.1 Let $y \in L^{1}[0,1] \cap C(0,1)$. Then the unique solution of the two-term $F B V P(1)$ is

$$
u(t)=\int_{0}^{1} G(t, s) y(s) d s
$$


where

$$
G(t, s)=\frac{1}{g(1)} \begin{cases}g(t) g(1-s), & 0 \leq t \leq s \leq 1 \\ g(t) g(1-s)-g(t-s) g(1), & 0 \leq s \leq t \leq 1\end{cases}
$$

Proof It follows from [33] that the general solution of the equation

$$
-D_{0+}^{\alpha} u(t)+a u(t)=y(t)
$$

can be expressed by

$$
u(t)=-\int_{0}^{t} g(t-s) y(s) d s+c_{1} g(t)+c_{2} g^{\prime}(t)+c_{3} g^{\prime \prime}(t) .
$$

By direct calculation, we have

$$
\begin{aligned}
& g^{\prime}(t)=\sum_{k=0}^{+\infty} \frac{a^{k} t^{(k+1) \alpha-2}}{\Gamma((k+1) \alpha-1)}>0, \quad \forall t>0, \\
& g^{\prime \prime}(t)=\sum_{k=0}^{+\infty} \frac{a^{k} t^{(k+1) \alpha-3}}{\Gamma((k+1) \alpha-2)}>0, \quad \forall t>0 .
\end{aligned}
$$

By $u(0)=u^{\prime}(0)=0$, there is $c_{3}=c_{2}=0$. Then we get

$$
u(1)=-\int_{0}^{1} g(1-s) y(s) d s+c_{1} g(1) .
$$

It follows from $u(1)=0$ that

$$
c_{1}=\frac{\int_{0}^{1} g(1-s) y(s) d s}{g(1)} .
$$

Therefore, the solution of (1) is

$$
\begin{aligned}
u(t) & =-\int_{0}^{t} g(t-s) y(s) d s+\frac{\int_{0}^{1} g(1-s) y(s) d s}{g(1)} g(t) \\
& =\frac{\int_{0}^{1} g(t) g(1-s) y(s) d s-\int_{0}^{t} g(1) g(t-s) y(s) d s}{g(1)} \\
& =\int_{0}^{1} G(t, s) y(s) d s .
\end{aligned}
$$

Remark 2.1 The unique solution given in Lemma 2.1 satisfies $u \in A C^{1}[0,1]$, where

$$
A C^{n}[0,1]=\left\{v:[0,1] \rightarrow \mathbf{R} \text { and } \frac{d^{n-1} v}{d t^{n-1}} \text { is absolutely continuous on }[0,1]\right\} .
$$

Proof In fact, we have

$$
u(t)=-\int_{0}^{t} g(t-s) y(s) d s+c_{1} g(t)=c_{1} g(t)-I_{0+}^{\alpha} y(t)-\sum_{k=1}^{+\infty} I_{0_{+}}^{(k+1) \alpha} y(t) .
$$


It follows from [32, Lemma 2.1] that $I_{0+}^{\alpha} y(t) \in A C^{1}[0,1]$ and

$$
I_{0+}^{(k+1) \alpha} y(t) \in A C^{[(k+1) \alpha]-1}[0,1] .
$$

Notice that $g(t) \in A C^{1}[0,1]$, we can get $u \in A C^{1}[0,1]$.

\section{Main results}

Lemma 3.1 For $0 \leq s \leq t \leq 1$, we have

$$
g_{1}(t) g_{1}(1-s) \geq g_{1}(t-s) g_{1}(1) .
$$

Proof For $t>0$, we have

$$
g_{1}^{\prime}(t)=\sum_{k=0}^{+\infty} \frac{(k \alpha+\alpha-2) a^{k} t^{k \alpha+\alpha-3}}{\Gamma(k \alpha+\alpha)}>0 .
$$

Therefore, $g_{1}(t)$ is strictly increasing on $[0,1]$. By direct calculation, we have

$$
g_{1}^{\prime \prime}(t)=t^{\alpha-4} h\left(a t^{\alpha}\right)<t^{\alpha-4} h\left(a^{*}\right)=0, \quad t \in(0,1),
$$

which implies $g_{1}^{\prime}(t)$ is strictly decreasing on $(0,1]$. Thus

$$
\begin{aligned}
& \frac{\partial}{\partial s}\left[g_{1}(t) g_{1}(1-s)-g_{1}(t-s) g_{1}(1)\right] \\
& \quad=g_{1}^{\prime}(t-s) g_{1}(1)-g_{1}(t) g_{1}^{\prime}(1-s) \\
& \quad \geq g_{1}^{\prime}(1-s)\left[g_{1}(1)-g_{1}(t)\right] \geq 0 .
\end{aligned}
$$

Therefore we can get

$$
g_{1}(t) g_{1}(1-s)-g_{1}(t-s) g_{1}(1) \geq 0,
$$

that is,

$$
g_{1}(t) g_{1}(1-s) \geq g_{1}(t-s) g_{1}(1) .
$$

Lemma 3.2 Assume that $s^{\star} \in(0,1)$ satisfies $s^{\star}=\left(1-s^{\star}\right)^{\alpha-2}$, then

$$
\min \left\{s,(1-s)^{\alpha-2}\right\} \leq \frac{s(1-s)^{\alpha-2}}{s^{\star}}, \quad s \in[0,1] .
$$

Proof It is clear that $k(s)$ is strictly decreasing on $[0,1]$. Notice that $k(0)=1$ and $k(1)=-1$, we know $k(s)$ has a unique root $s^{\star}$ on $(0,1)$, that is, $s^{\star}=\left(1-s^{\star}\right)^{\alpha-2}$. Therefore,

$$
\min \left\{s,(1-s)^{\alpha-2}\right\}= \begin{cases}s, & s \in\left[0, s^{\star}\right], \\ (1-s)^{\alpha-2}, & s \in\left[s^{\star}, 1\right] .\end{cases}
$$

Thus

$$
\min \left\{s,(1-s)^{\alpha-2}\right\} \leq \frac{s(1-s)^{\alpha-2}}{s^{\star}}, \quad s \in[0,1] .
$$


Theorem 3.1 The Green's function $G(t, s)$ satisfies the following properties:

$\left(p_{1}\right) G(t, s)>0, \forall t, s \in(0,1)$;

$\left(p_{2}\right) G(t, s)=G(1-s, 1-t), \forall t, s \in[0,1]$;

$\left(p_{3}\right) G(t, s) \geq M_{1} s(1-s)^{\alpha-1}(1-t) t^{\alpha-1}, \forall t, s \in[0,1]$;

$\left(p_{4}\right) G(t, s) \leq M_{2} s(1-s)^{\alpha-1}, \forall t, s \in[0,1]$, where

$$
M_{1}=\frac{1}{g(1)[\Gamma(\alpha)]^{2}}, \quad M_{2}=\frac{\left[g^{\prime}(1)\right]^{2}}{g(1) s^{\star}} .
$$

Proof Since $\left(p_{2}\right)$ is trivially true and $\left(p_{1}\right)$ can be derived from $\left(p_{3}\right)$, it remains to verify $\left(p_{3}\right)$ and $\left(p_{4}\right)$.

For $t \in[0,1]$, it is easy to check that

$$
g(t)=\sum_{k=0}^{+\infty} \frac{a^{k} t^{k \alpha+\alpha-1}}{\Gamma((k+1) \alpha)} \geq \frac{t^{\alpha-1}}{\Gamma(\alpha)}
$$

and

$$
g(t) \leq t^{\alpha-1} \sum_{k=0}^{+\infty} \frac{a^{k}}{\Gamma((k+1) \alpha)}=t^{\alpha-1} g(1)
$$

Combining the notations of $g$ and $g^{\prime}$ with

$$
\Gamma((k+1) \alpha)>\Gamma((k+1) \alpha-1), \quad k=0,1,2, \ldots,
$$

one has

$$
g(1)<g^{\prime}(1)
$$

Case (I): $0 \leq t \leq s \leq 1$.

By (6), one has

$$
\begin{aligned}
G(t, s) & =\frac{g(t) g(1-s)}{g(1)} \geq \frac{t^{\alpha-1}(1-s)^{\alpha-1}}{g(1)[\Gamma(\alpha)]^{2}} \\
& \geq M_{1} s(1-s)^{\alpha-1}(1-t) t^{\alpha-1} .
\end{aligned}
$$

By (7), one has

$$
\begin{aligned}
G(t, s) & \leq g(1) t^{\alpha-1}(1-s)^{\alpha-1} \\
& \leq g(1) s(1-s)^{\alpha-1} \\
& \leq M_{2} s(1-s)^{\alpha-1} .
\end{aligned}
$$

Case (II): $0<s<t<1$.

It is obvious that

$$
g(t)=\operatorname{tg}_{1}(t) .
$$


Therefore, it follows from Lemma 3.1 and (7) that

$$
\begin{aligned}
G(t, s) & =\frac{g(t) g(1-s)-g(t-s) g(1)}{g(1)} \\
& =\frac{t(1-s) g_{1}(t) g_{1}(1-s)-(t-s) g_{1}(t-s) g_{1}(1)}{g(1)} \\
& \geq \frac{g_{1}(t) g_{1}(1-s)[t(1-s)-(t-s)]}{g(1)} \\
& =\frac{g_{1}(t) g_{1}(1-s) s(1-t)}{g(1)} \\
& \geq \frac{t^{\alpha-2}(1-s)^{\alpha-2} s(1-t)}{g(1)[\Gamma(\alpha)]^{2}} \\
& \geq M_{1} s(1-s)^{\alpha-1}(1-t) t^{\alpha-1} .
\end{aligned}
$$

By the monotonicity of $g^{\prime}(t)$, we have

$$
\begin{aligned}
\frac{\partial}{\partial s} G(t, s) & =\frac{g^{\prime}(t-s) g(1)-g(t) g^{\prime}(1-s)}{g(1)} \\
& \leq \frac{g^{\prime}(1-s)[g(1)-g(t)]}{g(1)}
\end{aligned}
$$

By Lagrange's mean value theorem, there exist $\zeta \in(1-s, 1)$ and $\eta \in(t, 1)$ such that

$$
\begin{aligned}
G(t, s) & =\int_{0}^{s} \frac{\partial}{\partial \tau} G(t, \tau) d \tau \\
& \leq \int_{0}^{s} \frac{g^{\prime}(1-\tau)[g(1)-g(t)]}{g(1)} d \tau \\
& =\frac{[g(1)-g(1-s)][g(1)-g(t)]}{g(1)} \\
& =\frac{g^{\prime}(\zeta) s g^{\prime}(\eta)(1-t)}{g(1)} \\
& \leq \frac{\left[g^{\prime}(1)\right]^{2} s(1-s)}{g(1)} .
\end{aligned}
$$

On the other hand, it follows from (8) that

$$
G(t, s) \leq \frac{g(t) g(1-s)}{g(1)} \leq g(1) t^{\alpha-1}(1-s)^{\alpha-1} \leq g(1)(1-s)^{\alpha-1}
$$

Combining (13) and (14) with (6), we have

$$
\begin{aligned}
G(t, s) & \leq \min \left\{\frac{\left[g^{\prime}(1)\right]^{2} s(1-s)}{g(1)}, g^{\prime}(1)(1-s)^{\alpha-1}\right\} \\
& =\min \left\{\frac{g^{\prime}(1) s}{g(1)},(1-s)^{\alpha-2}\right\} \times g^{\prime}(1)(1-s) \\
& \leq \min \left\{s,(1-s)^{\alpha-2}\right\} \times \max \left\{1, \frac{g^{\prime}(1)}{g(1)}\right\} \times g^{\prime}(1)(1-s)
\end{aligned}
$$




$$
\begin{aligned}
& \leq \frac{s(1-s)^{\alpha-2}}{s^{\star}} \times \frac{g^{\prime}(1)}{g(1)} \times g^{\prime}(1)(1-s) \\
& =M_{2} s(1-s)^{\alpha-1}
\end{aligned}
$$

It follows from (10) and (12) that $\left(p_{3}\right)$ holds. On the other hand, (11) and (15) yield $\left(p_{4}\right)$ holds.

Corollary 3.1 It follows from $\left(p_{2}\right)$ and $\left(p_{4}\right)$ of Theorem 3.1 that

$$
G(t, s) \leq M_{2}(1-t) t^{\alpha-1}, \quad \forall t, s \in[0,1]
$$

\section{Applications}

\subsection{Semipositone problem}

In this section, we consider the existence and multiplicity of positive solutions to the semipositone FBVP:

$$
\left\{\begin{array}{l}
-D_{0+}^{\alpha} u(t)+a u(t)=\lambda f(t, u(t)), \quad 0<t<1, \\
u(0)=u^{\prime}(0)=0, \quad u(1)=0 .
\end{array}\right.
$$

For convenience, we list here the hypotheses to be used in this section:

$\left(H_{2}\right) f \in C((0,1) \times(0,+\infty),(-\infty,+\infty))$ and satisfies

$$
f(t, x) \geq-e(t), \quad(t, x) \in(0,1) \times(0,+\infty)
$$

where $e \in L^{1}[0,1] \cap C(0,1)$ is nonnegative and $\int_{0}^{1} e(s) d s>0$.

$\left(H_{3}\right)$ For any $R \geq r>0$, there exists $\Psi_{r, R} \in L^{1}[0,1] \cap C(0,1)$ such that

$$
f(t, x)+e(t) \leq \Psi_{r, R}(t), \quad \forall t \in(0,1), x \in\left[r(1-t) t^{\alpha-1}, R\right] .
$$

$\left(H_{4}\right)$ There exists $\left[c_{1}, d_{1}\right] \subset(0,1)$ such that

$$
\liminf _{x \rightarrow 0^{+}} \min _{t \in\left[c_{1}, d_{1}\right]} f(t, x)=+\infty
$$

$\left(H_{5}\right)$ There exists $\left[c_{2}, d_{2}\right] \subset(0,1)$ such that

$$
\liminf _{x \rightarrow+\infty} \min _{t \in\left[c_{2}, d_{2}\right]} \frac{f(t, x)}{x}=+\infty
$$

Remark 4.1 Condition $\left(H_{4}\right)$ implies that $f(t, x)$ is singular at $x=0$.

Let $E=C[0,1]$ be endowed with the maximum norm $\|u\|=\max _{0 \leq t \leq 1}|u(t)|$. Define a cone

$$
P=\left\{u \in E: u(t) \geq \frac{M_{1}\|u\|}{M_{2}}(1-t) t^{\alpha-1}, t \in[0,1]\right\} .
$$

Denote $B_{r}=\{u(t) \in E:\|u(t)\|<r\}$ and

$$
P_{r}=P \cap B_{r}
$$


Lemma 4.1 The unique solution of the FBVP

$$
\left\{\begin{array}{l}
-D_{0+}^{\alpha} u(t)+a u(t)=e(t), \quad 0<t<1 \\
u(0)=u^{\prime}(0)=u(1)=0
\end{array}\right.
$$

is

$$
\omega(t)=\int_{0}^{1} G(t, s) e(s) d s
$$

with

$$
\omega(t) \leq M_{2}(1-t) t^{\alpha-1} \int_{0}^{1} e(s) d s
$$

Proof The lemma can be deduced from Lemma 2.1 and Corollary 3.1, so we omit it.

Next we consider the auxiliary FBVP:

$$
\left\{\begin{array}{l}
-D_{0+}^{\alpha} u(t)+a u(t)=\lambda\left[f\left(t,[u(t)-\lambda \omega(t)]^{+}\right)+e(t)\right]=0, \quad 0<t<1, \\
u(0)=u^{\prime}(0)=u(1)=0
\end{array}\right.
$$

where $[u(t)-\lambda \omega(t)]^{+}=\max \{u(t)-\lambda \omega(t), 0\}$.

Let

$$
A u(t)=\int_{0}^{1} G(t, s)\left[f\left(s,[u(s)-\lambda \omega(s)]^{+}\right)+e(s)\right] d s .
$$

Lemma 4.2 Suppose that $\left(H_{2}\right)$ and $\left(H_{3}\right)$ hold. Then, for any $\lambda>0$ and

$$
r>\frac{\lambda M_{2}^{2} \int_{0}^{1} e(s) d s}{M_{1}}
$$

$A: P \backslash P_{r} \rightarrow P$ is completely continuous.

Proof For any $u \in P$ with $\|u\| \geq r$, one has

$$
u(t)-\lambda \omega(t) \geq\left[\frac{M_{1} r}{M_{2}}-\lambda M_{2} \int_{0}^{1} e(s) d s\right](1-t) t^{\alpha-1}>0, \quad \forall t \in(0,1) .
$$

The rest of the proof is similar to Lemma 2.6 in [21], we omit it here.

By the extension theorem of completely continuous operator (see [34]), there exists an extension operator $\widetilde{A}: P \rightarrow P$, which is still completely continuous. Without loss of generality, we still write it as $A$.

Lemma 4.3 ([34]) Let $E$ be a real Banach space, $P \subset E$ be a cone. Assume that $\Omega_{1}$ and $\Omega_{2}$ are two bounded open subsets of $E$ with $\theta \in \Omega_{1}, \bar{\Omega}_{1} \subset \Omega_{2}, A: P \cap\left(\bar{\Omega}_{2} \backslash \Omega_{1}\right) \rightarrow P$ is a completely continuous operator such that either 
(1) $\|A u\| \leq\|u\|, u \in P \cap \partial \Omega_{1}$ and $\|A u\| \geq\|u\|, u \in P \cap \partial \Omega_{2}$; or

(2) $\|A u\| \geq\|u\|, u \in P \cap \partial \Omega_{1}$ and $\|A u\| \leq\|u\|, u \in P \cap \partial \Omega_{2}$.

Then $A$ has a fixed point in $P \cap\left(\bar{\Omega}_{2} \backslash \Omega_{1}\right)$.

Theorem 4.1 Assume that $\left(H_{2}\right)-\left(H_{5}\right)$ hold. Then there exists $\lambda^{*}>0$ such that FBVP (16) has at least two positive solutions for any $\lambda \in\left(0, \lambda^{*}\right)$.

Proof For

$$
M^{\prime}=: \frac{2^{\alpha+1} M_{2}^{2} \int_{0}^{1} e(s) d s}{M_{1}^{2} \int_{c_{1}}^{d_{1}} s(1-s)^{\alpha-1} d s},
$$

$\left(H_{4}\right)$ guarantees there exists $X_{1} \in(0,1)$ such that

$$
f(t, x)>M^{\prime}, \quad \forall(t, x) \in\left[c_{1}, d_{1}\right] \times\left(0, X_{1}\right] .
$$

Let

$$
\lambda^{*}=\min \left\{\frac{M_{1} X_{1}}{2 M_{2}^{2} \int_{0}^{1} e(s) d s}, \frac{1+M_{2}}{M_{2} \int_{0}^{1} \Psi_{M_{1}, 1+M_{2}}(s) d s}\right\} .
$$

For any $\lambda \in\left(0, \lambda^{*}\right)$, let

$$
r_{1}=\frac{2 \lambda M_{2}^{2} \int_{0}^{1} e(s) d s}{M_{1}}
$$

It is clear that $r_{1}<X_{1}<1$.

$\forall u \in \partial P_{r_{1}}$, one has

$$
u(t)-\lambda \omega(t) \leq r_{1}<X_{1}
$$

and

$$
\begin{aligned}
u(t)-\lambda \omega(t) & \geq\left[\frac{M_{1} r_{1}}{M_{2}}-\lambda M_{2} \int_{0}^{1} e(s) d s\right](1-t) t^{\alpha-1} \\
& =\lambda M_{2} \int_{0}^{1} e(s) d s(1-t) t^{\alpha-1}
\end{aligned}
$$

Then

$$
\begin{aligned}
A u(t) & =\lambda \int_{0}^{1} G(t, s)\left[f\left(s,[u(s)-\lambda \omega(s)]^{+}\right)+e(s)\right] d s \\
& \geq \lambda M_{1}(1-t) t^{\alpha-1} \int_{c_{1}}^{d_{1}} s(1-s)^{\alpha-1} M^{\prime} d s \\
& =\frac{2^{\alpha+1} \lambda M_{2}^{2} \int_{0}^{1} e(s) d s}{M_{1}}(1-t) t^{\alpha-1} \\
& =2^{\alpha} r_{1}(1-t) t^{\alpha-1}
\end{aligned}
$$


which implies

$$
\|A u\|>\|u\|, \quad \forall u \in \partial P_{r_{1}} .
$$

Let $r_{2}=1+M_{2}$. For any $u \in \partial P_{r_{2}}$, one has

$$
u(t)-\lambda \omega(t) \leq r_{2}
$$

and

$$
\begin{aligned}
u(t)-\lambda \omega(t) & \geq\left[\frac{M_{1} r_{2}}{M_{2}}-\lambda M_{2} \int_{0}^{1} e(s) d s\right](1-t) t^{\alpha-1} \\
& \geq M_{1}(1-t) t^{\alpha-1}
\end{aligned}
$$

This and $\left(H_{3}\right)$ yield

$$
f\left(t,[u(t)-\lambda \omega(t)]^{+}\right)+e(t) \leq \Psi_{M_{1}, 1+M_{2}}(t) .
$$

Then

$$
\begin{aligned}
A u(t) & =\lambda \int_{0}^{1} G(t, s)\left[f\left(s,[u(s)-\lambda \omega(s)]^{+}\right)+e(s)\right] d s \\
& \leq \lambda M_{2}(1-t) t^{\alpha-1} \int_{0}^{1} \Psi_{M_{1}, 1+M_{2}}(s) d s \\
& <\lambda^{*} M_{2} \int_{0}^{1} \Psi_{M_{1}, 1+M_{2}}(s) d s \\
& \leq 1+M_{2}=r_{2} .
\end{aligned}
$$

Therefore

$$
\|A u\|<\|u\|, \quad \forall u \in \partial P_{r_{2}} .
$$

For

$$
M^{\prime \prime}=: \frac{2^{\alpha+1} M_{2}}{\lambda M_{1}^{2} c_{2}^{\alpha}\left(1-d_{2}\right)^{\alpha}\left(d_{2}-c_{2}\right)},
$$

$\left(H_{5}\right)$ guarantees there exists $X_{2}>r_{2}$ such that

$$
f(t, x)>M^{\prime \prime} x, \quad \forall(t, x) \in\left[c_{2}, d_{2}\right] \times\left[X_{2},+\infty\right) .
$$

Let

$$
r_{3}=1+\frac{L M_{2}}{M_{1}},
$$

where

$$
L=\frac{X_{2}}{c_{2}^{\alpha-1}\left(1-d_{2}\right)} .
$$


It is easy to see that

$$
r_{2}<r_{3}<\frac{2 L M_{2}}{M_{1}} \text {. }
$$

For any $u \in \partial P_{r_{3}}$, one has

$$
u(t)-\lambda \omega(t) \geq L(1-t) t^{\alpha-1} .
$$

Hence

$$
u(t)-\lambda \omega(t)>L\left(1-d_{2}\right) c_{2}^{\alpha-1}=X_{2}, \quad t \in\left[c_{2}, d_{2}\right]
$$

Thus

$$
\begin{aligned}
A u(t) & =\lambda \int_{0}^{1} G(t, s)\left[f\left(s,[u(s)-\lambda \omega(s)]^{+}\right)+e(s)\right] d s \\
& \geq \lambda M_{1}(1-t) t^{\alpha-1} \int_{c_{2}}^{d_{2}} s(1-s)^{\alpha-1} M^{\prime \prime} X_{2} d s .
\end{aligned}
$$

Then

$$
\begin{aligned}
\|A u\| & \geq \lambda M_{1}\left(\frac{1}{2}\right)^{\alpha} M^{\prime \prime} X_{2} \int_{c_{2}}^{d_{2}} s(1-s)^{\alpha-1} d s \\
& >\lambda M_{1}\left(\frac{1}{2}\right)^{\alpha} M^{\prime \prime} X_{2} c_{2}\left(1-d_{2}\right)^{\alpha-1}\left(d_{2}-c_{2}\right) \\
& =\frac{2 L M_{2}}{M_{1}}>r_{3},
\end{aligned}
$$

that is,

$$
\|A u\|>\|u\|, \quad \forall u \in P_{r_{3}} .
$$

Combining (18) - (20) with Lemma 4.3, we get $A$ has at least two fixed points $u_{1}$, $u_{2}$ with $r_{1}<\left\|u_{1}\right\|<r_{2}<\left\|u_{2}\right\|<r_{3}$, that is, $u_{1}$ and $u_{2}$ are solutions of the auxiliary FBVP (17). It is clear that $u_{i}(t)-\lambda \omega(t)>0$ on $(0,1), i=1,2$. Let $\bar{u}_{i}(t)=u_{i}(t)-\lambda \omega(t), i=1,2$. Then $\bar{u}_{1}(t)$ and $\bar{u}_{2}(t)$ are two positive solutions of the semipositone FBVP (16).

Corollary 4.1 Suppose that either $\left(H_{2}\right)-\left(H_{4}\right)$ or $\left(H_{2}\right),\left(H_{3}\right)$, and $\left(H_{5}\right)$ hold. Then FBVP $(16)$ has at least one positive solution provided $\lambda$ is small enough.

Example 4.1 Consider the following problem:

$$
\left\{\begin{array}{l}
-D_{0+}^{\frac{5}{2}} u(t)+\frac{1}{4} u(t)=\lambda f(t, u(t)), \quad 0<t<1 \\
u(0)=u^{\prime}(0)=0, \quad u(1)=0,
\end{array}\right.
$$

with

$$
f(t, x)=x^{2}+x^{-\frac{1}{2}}-t^{-\frac{1}{2}}(1-t)^{-\frac{1}{3}} .
$$


It is clear that $f(t, x)$ is singular at $t=0,1$, and $x=0$. For $x \in[0,+\infty)$, notice that $\Gamma(\cdot)$ is strictly increasing on $[2,+\infty)$, we have

$$
\begin{aligned}
h(x) & =-\frac{1}{3 \Gamma\left(\frac{1}{2}\right)}+\sum_{k=1}^{+\infty} \frac{x^{k}}{\left(\frac{5 k}{2}+\frac{3}{2}\right) \Gamma\left(\frac{5 k}{2}-\frac{1}{2}\right)} \\
& \leq-\frac{1}{3 \Gamma\left(\frac{1}{2}\right)}+\sum_{k=1}^{+\infty} \frac{x^{k}}{3 \Gamma\left(\frac{5 k}{2}-\frac{1}{2}\right)} \\
& \leq-\frac{1}{3 \sqrt{\pi}}+\frac{1}{3} \sum_{k=1}^{+\infty} \frac{x^{k}}{\Gamma(2 k)} \\
& =-\frac{1}{3 \sqrt{\pi}}+\frac{\sqrt{x}}{6}\left[e^{\sqrt{x}}-e^{-\sqrt{x}}\right] .
\end{aligned}
$$

By direct calculation, we have

$$
\frac{e^{\frac{1}{2}}-e^{-\frac{1}{2}}}{12}-\frac{1}{3 \sqrt{\pi}} \approx-0.1012136<0
$$

that is, $h\left(\frac{1}{4}\right)<0$. This yields $a^{*}>\frac{1}{4}$, so $\left(H_{1}\right)$ holds.

Let

$$
\begin{aligned}
& e(t)=t^{-\frac{1}{2}}(1-t)^{-\frac{1}{3}}, \\
& \Psi_{r, R}(t)=R^{2}+r^{-\frac{1}{2}}(1-t)^{-\frac{1}{2}} t^{-\frac{3}{4}}, \\
& {\left[c_{1}, d_{1}\right]=\left[c_{2}, d_{2}\right]=\left[\frac{1}{4}, \frac{3}{4}\right] .}
\end{aligned}
$$

It is easy to check that $\left(H_{2}\right)-\left(H_{5}\right)$ hold. Therefore Theorem 4.1 ensures that FBVP $(21)$ has at least two positive solutions provided $\lambda$ is small enough.

\subsection{Uniqueness results}

In this section, we consider the uniqueness results of positive solution to the singular FBVP:

$$
\left\{\begin{array}{l}
-D_{0+}^{\alpha} u(t)+a u(t)=f(t, u(t), u(t)), \quad 0<t<1 \\
u(0)=u^{\prime}(0)=0, \quad u(1)=0 .
\end{array}\right.
$$

For convenience, we assume that the following assumptions hold in the rest of this paper: $\left(H_{6}\right) f \in C((0,1) \times[0,+\infty) \times(0,+\infty) \rightarrow[0,+\infty)), f(t, x, y)$ is nondecreasing on $x$, nonincreasing on $y$, and there exists $\mu \in(0,1)$ such that

$$
f\left(t, r x, \frac{y}{r}\right) \geq r^{\mu} f(t, x, y), \quad \forall x, y>0, r \in(0,1) .
$$

$\left(H_{7}\right) 0<\int_{0}^{1} f\left(s,(1-s) s^{\alpha-1},(1-s) s^{\alpha-1}\right) d s<+\infty$. 
Remark 4.2 Inequality (23) is equivalent to

$$
f\left(t, \frac{x}{r}, r y\right) \leq r^{-\mu} f(t, x, y), \quad \forall x, y>0, r \in(0,1)
$$

Define a cone $Q$ by

$$
Q=\left\{u \in E: \exists l_{u}>0, \text { such that } l_{u}(1-t) t^{\alpha-1} \geq u(t) \geq \frac{M_{1}\|u\|}{M_{2}}(1-t) t^{\alpha-1}\right\}
$$

Define a mixed monotone operator $T$ by

$$
T(u, v)=\int_{0}^{1} G(t, s) f(s, u(s), v(s)) d s
$$

Set $Q_{1}=Q \backslash\{\theta\}$, where $\theta$ is the zero element of $E$.

Lemma 4.4 $T: Q_{1} \times Q_{1} \rightarrow Q_{1}$.

Proof For $u, v \in Q_{1}, \exists l_{u}, l_{v}>0$ such that

$$
\begin{aligned}
& l_{u}(1-t) t^{\alpha-1} \geq u(t) \geq \frac{M_{1}\|u\|}{M_{2}}(1-t) t^{\alpha-1}, \\
& l_{v}(1-t) t^{\alpha-1} \geq v(t) \geq \frac{M_{1}\|v\|}{M_{2}}(1-t) t^{\alpha-1} .
\end{aligned}
$$

Denote

$$
\delta=\min \left\{\frac{1}{l_{u}}, \frac{M_{1}\|v\|}{M_{2}}, \frac{1}{2}\right\} .
$$

It follows from Corollary 3.1 and Remark 4.2 that

$$
\begin{aligned}
T(u, v) & =\int_{0}^{1} G(t, s) f(s, u(s), v(s)) d s \\
& \leq M_{2}(1-t) t^{\alpha-1} \int_{0}^{1} f(s, u(s), v(s)) d s \\
& \leq M_{2}(1-t) t^{\alpha-1} \int_{0}^{1} f\left(s, l_{u}(1-s) s^{\alpha-1}, \frac{M_{1}\|v\|}{M_{2}}(1-s) s^{\alpha-1}\right) d s \\
& \leq M_{2}(1-t) t^{\alpha-1} \int_{0}^{1} f\left(s, \frac{(1-s) s^{\alpha-1}}{\delta}, \delta(1-s) s^{\alpha-1}\right) d s \\
& \leq \delta^{-\mu} M_{2}(1-t) t^{\alpha-1} \int_{0}^{1} f\left(s,(1-s) s^{\alpha-1},(1-s) s^{\alpha-1}\right) d s \\
& <+\infty .
\end{aligned}
$$

By $\left(p_{3}\right)$ and $\left(p_{4}\right)$ of Theorem 3.1, we have

$$
T(u, v)(t) \geq M_{1}(1-t) t^{\alpha-1} \int_{0}^{1} s(1-s)^{\alpha-1} f(s, u(s), v(s)) d s
$$


and

$$
T(u, v)(t) \leq M_{2} \int_{0}^{1} s(1-s)^{\alpha-1} f(s, u(s), v(s)) d s
$$

which implies

$$
T(u, v)(t) \geq \frac{M_{1}\|T(u, v)\|}{M_{2}}(1-t) t^{\alpha-1}
$$

This and (25) yield $T: Q_{1} \times Q_{1} \rightarrow Q_{1}$ is well defined.

Theorem 4.2 The singular FBVP (22) has a unique positive solution.

Proof Let $w \in Q_{1}$, it follows from Lemma 4.4 that $T(w, w) \in Q_{1}$. Then we can select $r_{0} \in$ $(0,1)$ such that

$$
r_{0}^{1-\mu} w \leq T(w, w) \leq r_{0}^{-(1-\mu)} w
$$

Set

$$
u_{n}=T\left(u_{n-1}, v_{n-1}\right), \quad v_{n}=T\left(v_{n-1}, u_{n-1}\right), \quad n=1,2, \ldots,
$$

where

$$
u_{0}=r_{0}^{\frac{1}{2}} w, \quad v_{0}=r_{0}^{-\frac{1}{2}} w
$$

It is easy to see that $u_{i}, v_{i} \in Q_{1}, i=0,1, \ldots$, and

$$
u_{0} \leq u_{1} \leq \cdots \leq u_{n} \leq \cdots \leq v_{n} \leq \cdots \leq v_{1} \leq v_{0} .
$$

It follows from (23) and (24) that

$$
\begin{aligned}
& u_{1}=T\left(r_{0}^{\frac{1}{2}} w, r_{0}^{-\frac{1}{2}} w\right) \geq r_{0}^{\frac{\mu}{2}} T(w, w), \\
& v_{1}=T\left(r_{0}^{-\frac{1}{2}} w, r_{0}^{\frac{1}{2}} w\right) \leq r_{0}^{-\frac{\mu}{2}} T(w, w) .
\end{aligned}
$$

Then we have

$$
u_{1} \geq r_{0}^{\mu} v_{1}
$$

By induction, we can get

$$
u_{n} \geq r_{0}^{\mu^{n}} v_{n}, \quad n=1,2, \ldots
$$

Therefore, (28) and (29) yield

$$
0 \leq u_{n+m}-u_{n} \leq v_{n}-u_{n} \leq\left(1-r_{0}^{\mu^{n}}\right) v_{n} \leq\left(1-r_{0}^{\mu^{n}}\right) v_{0}
$$


Then $\left\{u_{n}\right\}$ is a Cauchy sequence. Similarly, we can get $\left\{v_{n}\right\}$ is a Cauchy sequence. It follows from (28) that there exist $u^{*}, v^{*} \in Q_{1}$ such that $\left\{u_{n}\right\}$ and $\left\{v_{n}\right\}$ converge to $u^{*}$ and $v^{*}$ respectively. Moreover,

$$
u_{n} \leq u^{*} \leq v^{*} \leq v_{n}, \quad n=1,2, \ldots
$$

This and (29) imply that

$$
\left\|v^{*}-u^{*}\right\| \leq\left\|v_{n}-u_{n}\right\| \leq\left(1-r_{0}^{\mu^{n}}\right)\left\|v_{0}\right\|, \quad n=1,2, \ldots .
$$

Hence

$$
u^{*}=v^{*} \text {. }
$$

By (30), we have

$$
u_{n+1}=T\left(u_{n}, v_{n}\right) \leq T\left(u^{*}, v^{*}\right)=T\left(v^{*}, u^{*}\right) \leq T\left(v_{n}, u_{n}\right)=v_{n+1} \text {. }
$$

Let $n \rightarrow+\infty$, we get

$$
u^{*} \leq T\left(u^{*}, v^{*}\right)=T\left(v^{*}, u^{*}\right) \leq v^{*} .
$$

Then we have $u^{*}=T\left(u^{*}, u^{*}\right)$, that is, $u^{*}$ is a positive fixed point of $T$.

Next, we will show that the positive fixed point of $T$ is unique. In fact, if $u \neq u^{*}$ is a positive fixed point of $T$, by Lemma 4.4, we have $u \in Q_{1}$. Denote

$$
r_{1}=\sup \left\{r \in(0,1): r u^{*} \leq u \leq r^{-1} u^{*}\right\}
$$

It is clear that $r_{1} \in(0,1)$ and

$$
r_{1} u^{*} \leq u \leq r_{1}^{-1} u^{*}
$$

Then

$$
u=T(u, u) \geq T\left(r_{1} u^{*}, r_{1}^{-1} u^{*}\right) \geq r_{1}^{\mu} T\left(u^{*}, u^{*}\right)=r_{1}^{\mu} u^{*}
$$

and

$$
u=T(u, u) \leq T\left(r_{1}^{-1} u^{*}, r_{1} u^{*}\right) \leq r_{1}^{-\mu} T\left(u^{*}, u^{*}\right)=r_{1}^{-\mu} u^{*}
$$

Therefore,

$$
r_{1}^{\mu} u^{*} \leq u \leq r_{1}^{-\mu} u^{*}
$$

This contradicts with the definition of $r_{1}$ since $r_{1}^{\mu}>r_{1}$. Consequently, the positive fixed point of $T$ is unique, that is, FBVP (22) has a unique positive solution. 
Remark 4.3 The iterative sequence $\left\{u_{n}\right\}$ defined by (27) converges uniformly to the unique positive solution $u^{*}$. Moreover, we have the error estimation

$$
\left\|u_{n}-u^{*}\right\| \leq\left(1-r_{0}^{\mu^{n}}\right)\left\|v_{0}\right\|
$$

with the rate of convergence

$$
\left\|u_{n}-u^{*}\right\|=O\left(1-r_{0}^{\mu^{n}}\right)=O\left(\mu^{n}\right) .
$$

Example 4.2 Consider the following problem:

$$
\left\{\begin{array}{l}
-D_{0+}^{\frac{5}{2}} u(t)+\frac{1}{4} u(t)=f(t, u(t), u(t)), \quad 0<t<1, \\
u(0)=u^{\prime}(0)=0, \quad u(1)=0,
\end{array}\right.
$$

with

$$
f(t, x, y)=x^{\frac{1}{3}}+y^{-\frac{1}{3}}
$$

It follows from Example 4.1 that $h\left(\frac{1}{4}\right)<0$, that is, $\left(H_{1}\right)$ holds. Clearly, $\left(H_{5}\right)$ and $\left(H_{6}\right)$ hold. Then Theorem 4.2 ensures that FBVP (31) has a unique positive solution $u^{*}$.

By direct calculation, we have

$$
\begin{aligned}
s^{\star}= & \frac{\sqrt{5}-1}{2}, \\
g(1) & =\frac{1}{\Gamma\left(\frac{5}{2}\right)}+\sum_{k=1}^{+\infty} \frac{\left(\frac{1}{4}\right)^{k}}{\Gamma\left(\frac{5}{2} k+\frac{5}{2}\right)}<\frac{1}{\Gamma\left(\frac{5}{2}\right)}+\frac{\frac{1}{4}}{\Gamma(5)}+\sum_{k=2}^{+\infty} \frac{\left(\frac{1}{4}\right)^{k}}{\Gamma(2 k+2)} \\
& \approx 0.76339, \\
g(1)> & \frac{1}{\Gamma\left(\frac{5}{2}\right)}+\frac{\frac{1}{4}}{\Gamma(5)} \approx 0.76286, \\
g^{\prime}(1) & =\frac{1}{\Gamma\left(\frac{3}{2}\right)}+\sum_{k=1}^{+\infty} \frac{\left(\frac{1}{4}\right)^{k}}{\Gamma\left(\frac{5}{2} k+\frac{3}{2}\right)}<\frac{1}{\Gamma\left(\frac{3}{2}\right)}+\sum_{k=1}^{+\infty} \frac{\left(\frac{1}{4}\right)^{k}}{\Gamma(2 k+2)} \\
& \approx 1.17086 .
\end{aligned}
$$

Therefore,

$$
M_{1}>0.741278, \quad M_{2}<2.90772 .
$$

Let

$$
w(t)=(1-t) t^{\frac{3}{2}} .
$$


By Theorem 3.1 and Corollary 3.1, we have

$$
\begin{aligned}
& T(\omega, \omega) \leq M_{2}\left[B\left(\frac{4}{3}, \frac{3}{2}\right)+B\left(\frac{2}{3}, \frac{1}{2}\right)\right] \times w \leq 8.8541 \times w \\
& T(\omega, \omega) \geq M_{1}\left[B\left(\frac{17}{6}, \frac{5}{2}\right)+B\left(\frac{13}{6}, \frac{3}{2}\right)\right] \times w \geq 0.2196 \times w .
\end{aligned}
$$

Set

$$
\begin{aligned}
& r_{0}=\frac{1}{27}, \quad u_{0}=\frac{w}{3 \sqrt{3}}, \quad v_{0}=3 \sqrt{3} w \\
& u_{n}=T\left(u_{n-1}, v_{n-1}\right), \quad v_{n}=T\left(v_{n-1}, u_{n-1}\right), \quad n=1,2, \ldots
\end{aligned}
$$

Then (26) holds and $\left\|v_{0}\right\|=\frac{54}{25 \sqrt{5}} \approx 0.966$. Moreover,

$$
\left\|u_{n}-u^{*}\right\| \leq 0.966 \times\left(1-3^{-3^{1-n}}\right), \quad n=1,2, \ldots
$$

Then we have the rate of convergence

$$
\left\|u_{n}-u^{*}\right\|=O\left(\left(\frac{1}{3}\right)^{n}\right),
$$

and the error estimation

$$
\begin{gathered}
\left\|u_{1}-u^{*}\right\|<0.643999 \\
\left\|u_{2}-u^{*}\right\|<0.296213 \\
\left\|u_{3}-u^{*}\right\|<0.111005 \\
\left\|u_{4}-u^{*}\right\|<0.038517 \\
\left\|u_{5}-u^{*}\right\|<0.013014 \\
\left\|u_{6}-u^{*}\right\|<0.004358
\end{gathered}
$$

\section{Conclusions}

In this paper, we establish some positive properties of the Green's function for a class of FBVPs. The interesting point is that the linear operator of the FBVPs contains two terms. As application of the main results, we investigate the existence and multiplicity results of positive solutions for an FBVP under conditions that the nonlinearity may change sign and possess singularity, and we also consider the uniqueness results of positive solution for a singular FBVP.

\section{Acknowledgements}

The author would like to thank the referees for their pertinent comments and valuable suggestions.

\section{Funding}

This work was supported by the Natural Science Foundation of Shandong Province of China (ZR2017MA036); the National Natural Science Foundation of China (11871302); a Project of Shandong Province Higher Educational Science and

Technology Program (J18KA217), and the International Cooperation Program of Key Professors by Qufu Normal University. 
Abbreviations

FBVP, Fractional differential equations boundary value problem; FDEs, fractional differential equations.

\section{Availability of data and materials}

Data sharing not applicable to this article as no datasets were generated or analysed during the current study.

\section{Competing interests}

The author declares that there is no conflict of interests regarding the publication of this paper.

\section{Authors' contributions}

The author read and approved the final manuscript.

\section{Publisher's Note}

Springer Nature remains neutral with regard to jurisdictional claims in published maps and institutional affiliations.

Received: 10 December 2019 Accepted: 14 February 2020 Published online: 21 February 2020

\section{References}

1. Bagley, R., Torvik, P.: On the appearance of the fractional derivative in the behaviour of real materials. J. Appl. Mech. 51, 294-298 (1984)

2. Metzler, R., Klafter, J.: Boundary value problems for fractional diffusion equations. Physica A 278, 107-125 (2000)

3. Diethelm, K., Luchko, Y.: Numerical solution of linear multi-term initial value problems of fractional order. J. Comput. Anal. Appl. 6, 243-263 (2004)

4. Ford, N., Connolly, J.: Systems-based decomposition schemes for the approximate solution of multi-term fractional differential equations. Comput. Appl. Math. 229, 382-391 (2009)

5. Elshehawey, E., Elbarbary, E., Afifi, N., El-Shahed, M.: On the solution of the endolymph equation using fractiona calculus. Appl. Math. Comput. 124, 337-341 (2001)

6. Čermák, J., Kisela, T.: Stability properties of two-term fractional differential equations. Nonlinear Dyn. 80, 1673-1684 (2015)

7. Daftardar-Gejii, V., Bhalekar, S.: Boundary value problems for multi-term fractional differential equations. J. Math. Anal. Appl. 345, 754-765 (2008)

8. Ahmad, B., Ntouyas, S.: Existence results for a coupled system of Caputo type sequential fractional differential equations with nonlocal integral boundary conditions. Appl. Math. Comput. 266, 615-622 (2015)

9. Ahmad, B., Luca, R.: Existence of solutions for sequential fractional integro-differential equations and inclusions with nonlocal boundary conditions. Appl. Math. Comput. 339, 516-534 (2018)

10. Cabada, A., Wang, G.: Positive solutions of nonlinear fractional differential equations with integral boundary value conditions. J. Math. Anal. Appl. 389, 403-411 (2012)

11. Cabada, A., Hamdi, Z.: Nonlinear fractional differential equations with integral boundary value conditions. Appl. Math Comput. 228, 251-257 (2014)

12. Cui, Y.: Uniqueness of solution for boundary value problems for fractional differential equations. Appl. Math. Lett. 51 48-54 (2016)

13. Cui, Y., Ma, W., Sun, Q., Su, X.: New uniqueness results for boundary value problem of fractional differential equation. Nonlinear Anal., Model. Control 23, 31-39 (2018)

14. Jiang, D., Yuan, C.: The positive properties of the Green function for Dirichlet-type boundary value problems of nonlinear fractional differential equations and its application. Nonlinear Anal., Theory Methods Appl. 72, 710-719 (2010)

15. Hao, X., Wang, H.: Positive solutions of semipositone singular fractional differential systems with a parameter and integral boundary conditions. Open Math. 16, 581-596 (2018)

16. Henderson, J., Luca, R.: Existence of positive solutions for a singular fractional boundary value problem. Nonlinear Anal., Model. Control 22,99-114 (2017)

17. Su, X., Zhang, S.: Unbounded solutions to a boundary value problem of fractional order on the half-line. Comput. Math. Appl. 61, 1079-1087 (2011)

18. Wang, Y., Liu, L., Wu, Y.: Positive solutions for a class of fractional boundary value problem with changing sign nonlinearity. Nonlinear Anal., Theory Methods Appl. 74, 6434-6441 (2011)

19. Wang, Y., Liu, L., Wu, Y.: Existence and uniqueness of a positive solution to singular fractional differential equations. Bound. Value Probl. 2012, Article ID 81 (2012)

20. Wang, Y., Liu, L.: Positive properties of the Green function for two-term fractional differential equations and its application. J. Nonlinear Sci. Appl. 10, 2094-2102 (2017)

21. Wang, Y.: Existence and multiplicity of positive solutions for a class of singular fractional nonlocal boundary value problems. Bound. Value Probl. 2019, Article ID 92 (2019)

22. Wang, Y.: Necessary conditions for the existence of positive solutions to fractional boundary value problems at resonance. Appl. Math. Lett. 97, 34-40 (2019)

23. Wang, G., Ahmad, B., Zhang, L.: Existence results for nonlinear fractional differential equations with closed boundary conditions and impulses. Adv. Differ. Equ. 2012, Article ID 169 (2012)

24. Zhang, H., Li, Y., Xu, J.: Positive solutions for a system of fractional integral boundary value problems involving Hadamard-type fractional derivatives. Complexity 2019, Article ID 2671539 (2019)

25. Zhang, X., Liu, L., Wiwatanapataphee, B., Wu, Y.: The eigenvalue for a class of singular p-Laplacian fractional differential equations involving the Riemann-Stieltjes integral boundary condition. Appl. Math. Comput. 235, 412-422 (2014)

26. Zhang, X., Liu, L., Wu, Y.: The uniqueness of positive solution for a fractional order model of turbulent flow in a porous medium. Appl. Math. Lett. 37, 26-33 (2014) 
27. Zhang, X., Shao, Z., Zhong, Q., Zhao, Z.: Triple positive solutions for semipositone fractional differential equations m-point boundary value problems with singularities and p-q-order derivatives. Nonlinear Anal., Model. Control 23, 889-903 (2018)

28. Zhang, X., Zhong, Q.: Triple positive solutions for nonlocal fractional differential equations with singularities both on time and space variables. Appl. Math. Lett. 80, 12-19 (2018)

29. Zhu, B., Liu, L., Wu, Y.: Local and global existence of mild solutions for a class of semilinear fractional integro-differential equations. Fract. Calc. Appl. Anal. 20, 1338-1355 (2017)

30. $\mathrm{Xu}, \mathrm{X}$., Fei, X.: The positive properties of Green's function for three point boundary value problems of nonlinear fractional differential equations and its applications. Commun. Nonlinear Sci. Numer. Simul. 17, 1555-1565 (2012)

31. Zou, Y., He, G.: On the uniqueness of solutions for a class of fractional differential equations. Appl. Math. Lett. 74, 68-73 (2017)

32. Staněk, S.: The existence of positive solutions of singular fractional boundary value problems. Comput. Math. Appl. 62, 1379-1388 (2011)

33. Podlubny, I.: Fractional Differential Equations. Academic Press, San Diego (1999)

34. Guo, D.: Nonlinear Functional Analysis. Shandong Science and Technology Press, Jinan (1985) (in Chinese)

\section{Submit your manuscript to a SpringerOpen ${ }^{\mathcal{O}}$} journal and benefit from:

- Convenient online submission

- Rigorous peer review

- Open access: articles freely available online

- High visibility within the field

- Retaining the copyright to your article

Submit your next manuscript at $\boldsymbol{~ s p r i n g e r o p e n . c o m ~}$ 